\title{
Percepção dos profissionais de saúde quanto a gestão dos resíduos de serviço de saúde
}

\begin{abstract}
A gestão de resíduos de serviço de saúde (RSS) constitui um grande desafio para o poder público, apesar dos avanços nos aspectos legislativos. O objetivo desta pesquisa foi avaliar a percepção dos profissionais de saúde, acerca da gestão de resíduos de serviço saúde, no Hospital Geral e Unidades Básicas de saúde em um município da Zona da Mata de Pernambuco. Para esta pesquisa foi realizada uma avaliação quantitativa e qualitativa, exploratória e descritiva. Trata-se de um estudo de caso com referência: 01 hospital geral (enfermeiros e auxiliares de enfermagem), 05 unidades básicas de saúde (enfermeiros, auxiliares de enfermagem, dentistas e ASB auxiliares de saúde bucal), com atendimento médico, odontológico e enfermagem. Os resultados buscaram mostrar a percepção dos profissionais envolvidos no serviço público de saúde em relação a gestão dos resíduos, nas etapas de segregação, acondicionamento, armazenamento, coleta e transporte e destinação final. Os resultados desenham o desafio nas instituições de saúde quanto a educação permanente, com abordagem sobre PGRSS, segurança no trabalho e meio ambiente. Em relação ao Plano de Gerenciamento de Resíduos de Serviço de Saúde (PGRSS), concluiu-se que 47\% dos funcionários desconhece a existência do documento elaborado pelo munícipio, contudo, 35\% disseram existir um Plano de Gerenciamento, embora este não tenha sido apresentado durante o desenvolvimento da pesquisa. No âmbito geral, o profissional de saúde, do serviço público do município da Zona da Mata de Pernambuco: Hospital Geral e Unidades Básicas de Saúde têm conhecimento sobre gestão de resíduos de serviços de saúde de acordo com as exigências da RDC n²22/2018 em torno de $54 \%$ do total.
\end{abstract}

Palavras-chave: Meio ambiente; Saúde pública; Resíduos sólidos.

\section{Health professionals' perception of health service waste management}

\begin{abstract}
Preservation Healthcare waste management is a major challenge for the public authorities, despite advances in legislative aspects. The objective of this research was to evaluate the perception of health professionals about the management of health care waste at the General Hospital and Basic Health Units in a municipality of Zona da Mata de Pernambuco. For this research a quantitative and qualitative, exploratory and descriptive evaluation was performed. This is a case study with reference: 01 general hospital (nurses and nursing assistants), 05 basic health units (nurses, nursing assistants, dentists) and oral health assistants with medical, dental and nursing care. The results sought to show the perception of the professionals involved in the public health service regarding waste management, in the stages of segregation, conditioning, storage, collection and transportation and final destination. The results draw the challenge in health institutions regarding continuing education, with an approach on Health Care Waste Management Plan, occupational safety and the environment Regarding the Health Care Waste Management Plan, it was concluded that $47 \%$ of the employees do not know the existence of the document prepared by the municipality, however, $35 \%$ said there was a Management Plan, although it was not presented during the development of the research. In the general context, the public sector health professional from the municipality of Zona da Mata de Pernambuco: General Hospital and Basic Health Units has knowledge about health services waste management in accordance with the requirements of Resolution of the Collegiate Board of the National Health Surveillance Agency of Brazil, RDC 222/2018 around 54\% of the total.
\end{abstract}

Keywords: Environment; Public health; Solid waste.

Topic: Engenharia Ambiental

Reviewed anonymously in the process of blind peer.

Paula Montenegro Gonçalves de Alencar Barros Faculdade de Tecnologia de Sete Lagoas, Brasil http://lattes.cnpq.br/3083197730081820 paulamgalencar@hotmail.com

Daniele de Castro Pessoa de Melo (iD) Instituto de Tecnologia de Pernambuco, Brasil http://lattes.cnpq.br/4010783198064867 http://orcid.org/0000-0003-4058-092X daniele.castro@itep.br

Eduardo Antônio Maia Lins (iD Instituto de Tecnologia de Pernambuco, Brasil http://lattes.cnpq.br/6148771863554184 http://orcid.org/0000-0002-9108-4179 eduardomaialins@gmail.com
Received: 05/12/2019

Approved: 12/01/2020

\author{
Ronaldo Faustino da Silva (iD \\ Instituto de Tecnologia de Pernambuco, Brasil \\ http://lattes.cnpq.br/7265569987870357 \\ http://orcid.org/0000-0001-8097-9420 \\ ronaldofaustino@recife.ifpe.edu.br
}

Referencing this:

BARROS, P. M. G. A.; MELO, D. C. P.; LINS, E. A. M.; SILVA, R. F. Percepção dos profissionais de saúde quanto a gestão dos resíduos de serviço de saúde. Revista Ibero Americana de Ciências Ambientais, v.11, n.1, p.201-210, 2020. DOI: http://doi.org/10.6008/CBPC2179$\underline{6858.2020 .001 .0019}$

DOI: 10.6008/CBPC2179-6858.2020.001.0019 


\section{INTRODUÇÃO}

Dentre os problemas ambientais que assolam a humanidade, a produção de resíduos constitui uma das principais formas de degradação do meio ambiente. Segundo Khobragade (2019), a profissão de saúde é uma das profissões mais admiráveis, destinadas a proteger os seres humanos contra doenças, restaurar a saúde e salvar vidas. Porém, os serviços de saúde são considerados uma das fontes de resíduos que provam ser uma ameaça ao meio ambiente e à saúde humana quando descartados inadequadamente.

Resíduos de Serviços de Saúde são os resíduos gerados por centros e pessoas que fornecem cuidados à saúde, incluindo produtos químicos, produtos farmacêuticos vencidos e itens radioativos, objetos perfurocortantes e infecciosos. Esses itens podem ser tóxicos, patogênicos e ambientalmente adversos devido à sua natureza não biodegradável e/ou reutilizável (KHOBRAGADE, 2019).

A importância de uma conduta ambiental pelas instituições de saúde se torna evidente frente aos dados da Associação Brasileira de Empresas de Limpeza Pública e Resíduos Especiais (ABRELPE), a qual afirma que a geração de resíduos Urbanos no Brasil é de 256.941 toneladas por dia, revelando um total anual de 78,4 milhões de toneladas. O montante coletado em 2017 registra um índice de cobertura de coleta em 91,2\% para o país, o que evidencia que 6,9 milhões de toneladas são resíduos que não foram objeto de coleta e, consequentemente, tiveram destino impróprio (ABRELPE, 2018).

Apenas cerca de 42,3 milhões de toneladas de RSU, ou 59,1\% do coletado, foram dispostos em aterros sanitários. O restante, que corresponde a $40,9 \%$ dos resíduos coletados, foi despejado em locais inadequados (ABRELPE, 2018). Dentre muitos resíduos, merecem destaque os Resíduos de Serviços de Saúde (RSS) que, apesar de representarem uma pequena parcela da totalidade de resíduos sólidos gerados, cerca de 1 a 3\%, oferecem um preocupante risco quando gerenciados inadequadamente, por serem possíveis fontes de propagação de doenças e degradação ambiental (ALVES et al., 2016).

No ano de 2017, apenas 4.518 municípios prestaram os serviços de coleta, tratamento e disposição final dos resíduos de saúde, 256.941 toneladas, o equivalente a 1,2 kg por habitante/ano (ABRELPE, 2018). A legislação brasileira estabelece aos geradores de RSS que determinadas classes de resíduos de serviços de saúde demandam o tratamento previamente à sua disposição final; no entanto, ainda cerca de $27,5 \%$ dos municípios brasileiros destinaram seus RSS sem declarar o tratamento prévio dado aos mesmos (ABRELPE, 2018).

Definem-se como geradores de Resíduos de Serviços de Saúde (RSS) todos os serviços cujas atividades estejam relacionadas com a atenção à saúde humana ou animal, inclusive os serviços de assistência domiciliar (laboratórios analíticos de produtos para saúde e necrotérios), drogarias, farmácias, unidades móveis de atendimento à saúde, dentre outros afins (ANVISA, 2018).

A regulamentação da questão dos Resíduos de Serviços de Saúde está a cargo da Agência Nacional de Vigilância Sanitária (ANVISA) e do Conselho Nacional do Meio Ambiente (CONAMA). Tais instituições assumem, no Brasil, o papel de orientar, definir regras e regular a conduta dos diferentes agentes no que diz respeito à geração e ao manejo dos RSS (BRASIL, 2014). 
No ano de 1993, a geração e o manejo dos RSS receberam a devida importância tendo em vista a publicação da Resolução CONAMA no 005/93, a qual definiu a obrigatoriedade dos serviços de saúde de elaborar o Plano de Gerenciamento de seus resíduos. Já no ano de 2004, entra em vigor a Resolução da Diretoria Colegiada - RDC n³06/2004 e a Lei $n^{\circ} 12.305 / 2010$, que veio a instituir a Política Nacional de Resíduos Sólidos (PNRS).

No ano de 2018 foi publicada uma nova normativa que abrange as novidades legais e novas tecnologias: A Resolução da Diretoria Colegiada (RDC) no 222/2018 da Agência Nacional de Vigilância Sanitária (ANVISA), que dispõe sobre os requisitos de Boas Práticas de Gerenciamento dos Resíduos de Serviços de Saúde, a RDC e a Resolução no 358/2005 do Conselho Nacional de Meio Ambiente (CONAMA), que definem os RSS como todo resíduo gerado em qualquer serviço prestador de assistência médica humana ou animal, e classifica-os em cinco grupos: grupo A - biológicos; grupos B - químicos; grupo C - radioativos; grupo D - comuns e grupo E perfurocortantes (ANVISA, 2018).

Com base nas normas supracitadas, os estabelecimentos de saúde que geram resíduos devem estabelecer um Plano de Gerenciamento, descrevendo as etapas de segregação, acondicionamento, coleta, armazenamento, transporte, tratamento, e disposição final, com objetivo de minimizar a produção e proporcionar aos resíduos gerados um encaminhamento seguro, visando à proteção dos funcionários, a preservação da saúde pública, dos recursos naturais e do meio ambiente (GOMES et al., 2015).

Os estabelecimentos de cuidados com a saúde humana, hospitais e unidades de saúde, produzem uma variedade de resíduos (alimentos, medicamentos, produtos químicos, equipamentos, instrumentos cirúrgicos etc.) enquanto tratam de pacientes externos e internados (ALAM et al., 2019). O Plano de Gerenciamento de Serviço de Saúde deve ser ajustado com as normas federais, estaduais e municipais e em consonância com processos institucionais de biossegurança relativos à coleta, transporte e disposição final. A implantação do PGRSS é responsabilidade do estabelecimento gerador (ANVISA, 2018).

Logo, o objetivo desta pesquisa foi avaliar o conhecimento e a percepção dos servidores da área de saúde acerca dos resíduos de saúde no Hospital Geral e Unidades Básicas de saúde do município de Cortês, na Zona da Mata de Pernambuco, pois se trata de uma região que atende muitos pacientes tanto da própria cidade quanto de cidades vizinhas para tratamento odontológico, clínico e hospitalar.

\section{METODOLOGIA}

O presente estudo foi realizado em um município da Zona da Mata do Estado de Pernambuco, tratase de um estudo de caso, pesquisas que tratam de um caso particular com finalidade de transformação, tomando como referência as Unidades Básicas de Saúde e o Hospital do município, com atendimento médico, odontológico e enfermagem.

A escolha do município na Zona da Mata/PE se deu em razão do hospital e suas unidades de saúdes prestarem atendimento não apenas a população local do município em estudo, mas também as pessoas advindas de cidades circunvizinhas, devido aos diversos tipos de procedimentos médico hospitalares que são oferecidos, acarretando consequentemente uma produção acentuada de resíduos devido à demanda de 
atendimento. Outro fator determinante se refere à autorização para realização desta pesquisa por parte da secretaria de saúde do município, na pessoa do seu Gestor.

Este estudo consistiu em uma pesquisa quantitativa exploratório-descritiva (levantamento de informações), em que foram utilizadas as seguintes técnicas de amostragem de dados: aplicação de questionário estruturado com questões fechadas. A seleção das unidades participantes seguiu critérios de conveniência (aceite do responsável para realização do estudo) contemplando as seguintes categorias de prestadores de serviços à saúde nas 5 unidades de saúde e no hospital geral. Foram avaliados profissionais nas áreas de enfermagem (enfermeiros e técnicos de enfermagem) e odontológicos (dentistas e assistente de saúde bucal-ASB) no hospital e nas 05 unidades básicas do município, descritos na Tabela 1.

Tabela 1: Distribuição e números de profissionais avaliados no questionário aplicado na pesquisa.

\begin{tabular}{|l|l|l|l|l|}
\hline \multirow{2}{*}{ Categoria } & No de Profissionais & Téc. De Enfermagem & Dentista & ASB* \\
\cline { 2 - 5 } & Enfermeiros & 09 & - & - \\
\hline Hospital & 07 & 01 & 01 & 01 \\
\hline Unidade A & 01 & 01 & 01 & 01 \\
\hline Unidade B & - & 01 & 01 & 01 \\
\hline Unidade C & 01 & 01 & 01 & 01 \\
\hline Unidade D & 01 & 01 & 01 & 01 \\
\hline Unidade E & - & \multicolumn{3}{l}{} \\
\hline
\end{tabular}

*Assistente de cirurgião dentista

A determinação amostral por atributos foi realizada utilizando a Equação 1.

$$
\mathrm{n}=\frac{\mathrm{N} \cdot \mathrm{p} \cdot \mathrm{q} \cdot\left(\mathrm{Z}_{\alpha / 2}\right)^{2}}{\mathrm{p} \cdot \mathrm{q} \cdot\left(\mathrm{Z}_{\alpha / 2}\right)^{2}+(\mathrm{N}-1) \cdot \mathrm{E}^{2}}
$$

Onde: $\mathrm{n}=$ número de indivíduos na amostra; $\mathrm{Z} \alpha / 2$ = valor crítico que corresponde ao grau de confiança desejado; $\mathrm{p}=$ proporção de sucesso $q=1-$ p e E = margem de erro para um grau de confiabilidade de 92\% (LEVIN, 1987; TRIOLA, 2008). Para cálculo de $\mathrm{n}$ foi considerado $\mathrm{N}=43, \mathrm{p}=0.92, \mathrm{q}=0.08, \mathrm{Z} \alpha / 2=1.75$ e $\mathrm{E}=0.04$. Então o número de entrevistados foi de 34 pessoas.

O instrumento de coleta de dados foi composto por questionário com questões fechadas, conduzido por único pesquisador, no período entre maio a julho de 2019 e entregue pessoalmente a todos os funcionários envolvidos, a fim de verificar a percepção dos profissionais de saúde no processo de gestão dos Resíduos de Serviços de Saúde do hospital e unidades básicas de saúde. Os formulários da pesquisa foram elaborados buscando englobar as principais informações sobre os procedimentos gerenciais e operacionais relativos às etapas de segregação, acondicionamento, coleta, armazenamentos interno e externo, transporte, tratamento e disposição final, adotando como referência a RDC no 222/2018 da Agência Nacional de Vigilância Sanitária (ANVISA, 2018).

\section{RESULTADOS E DISCUSSÃO}

O resultado por área mostra que $82 \%$ dos entrevistados têm conhecimento como realizar de maneira correta o manejo na segregação dos resíduos de saúde, 43\% o acondicionamento destes, $67 \%$ sabem como realizar corretamente a coleta e transporte, $62 \%$ responderam de forma correta sobre a destinação final. De acordo com o questionário realizado, foi possível observar que a gestão dos resíduos de serviço de saúde do hospital geral e unidades básicas de saúde estão com $54 \%$ de concordância em relação às normas 
preestabelecidas.

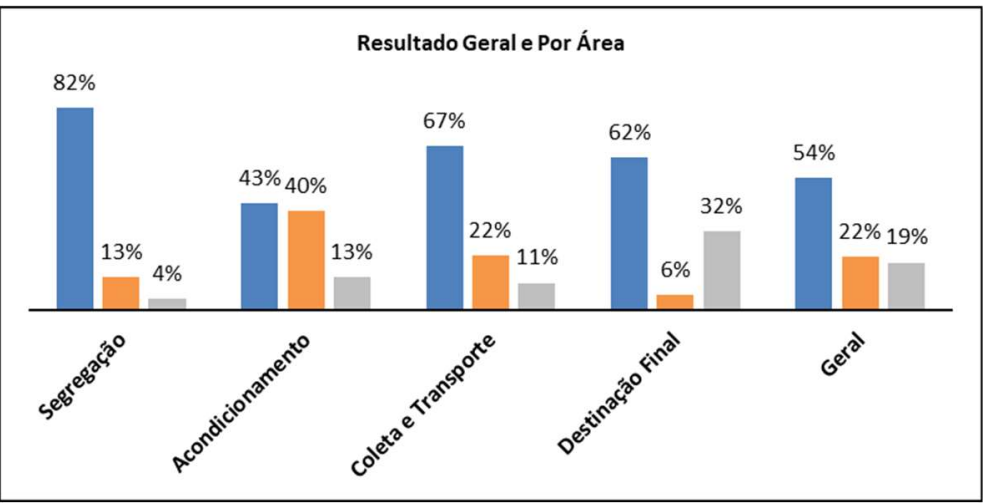

Figura 2: Visão geral sobre a percepção dos profissionais acerca da Gestão dos Resíduos de Serviço de Saúde.

Quanto ao conhecimento sobre a existência do Plano de Gerenciamento de Resíduos de Serviço de Saúde (PGRSS) no hospital e unidades básicas de saúde, no qual 35\% responderam (que existe um plano de gerenciamento), $18 \%$ responderam (que não existe plano ou está em implantação) e $47 \%$ responderam (que desconhece). Contudo, um plano de gerenciamento não foi apresentado pela gestão durante as entrevistas. De acordo com RDC 222/2018, artigo $5^{\circ}$, todo serviço gerador deve dispor de um Plano de Gerenciamento de RSS (PGRSS), observando as regulamentações federais municipais ou do Distrito Federal.

A carência do PGRSS em vários estabelecimentos não reflete apenas a atual gestão dos resíduos no município da Zona da Mata de Pernambuco, mas de outras regiões brasileiras. Estudos semelhantes realizados no Noroeste de São Paulo no serviço público, mostram que $29,4 \%$ dos profissionais não sabem o que significa o PGRSS e $11,8 \%$ relatam que não foi ou não sabem se foi implementado o PGRSS no estabelecimento em que trabalham 35,3\% não conhecem a legislação que versa sobre os RSS (GOMES et al., 2015).

Em contrapartida em outra localidade trabalhos de Szczerbowski et al. (2017) relatam que o Manejo de Resíduos de Serviço de Saúde realizado na Cidade de estrela do Sul/MG se constatou que as unidades de Saúde possuem o PGRSS e presta treinamentos semestrais acerca do manuseio adequado de resíduos sólidos a todos os profissionais de saúde, mantendo tanto os responsáveis pela saúde quanto a população em geral protegida de riscos decorrentes do manejo inadequado.

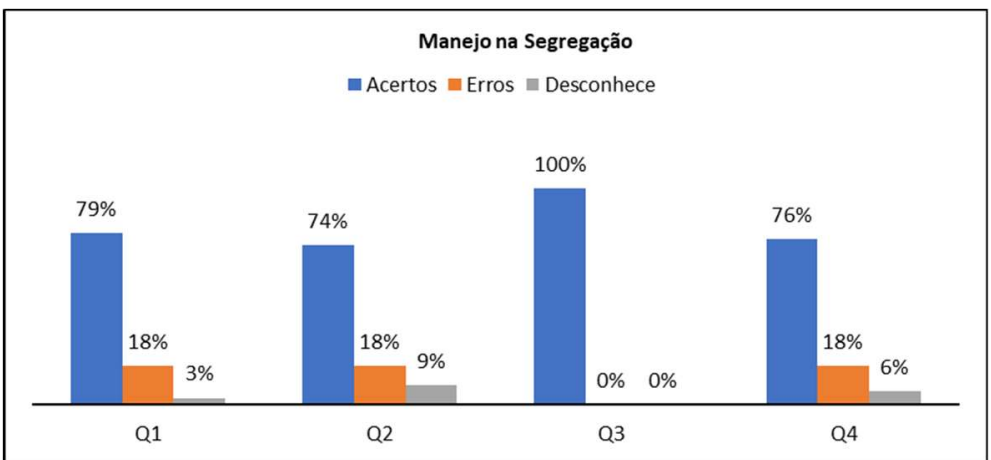

Figura 3: Percentual do conhecimento dos profissionais no manejo da segregação dos resíduos de serviços de saúde.

Segundo Cafure et al. (2015) os resíduos de Serviço de Saúde (RSS) são geradores de alto impacto ambiental causado pelo manejo e destinação inadequados, relacionando-se a contaminações dos lençóis 
freáticos. Isso demostra a importância de elaborar, implementar e desenvolver o Plano de Gerenciamento dos Resíduos de Serviços de Saúde.

Quanto à conformidade relacionada ao manejo na segregação dos resíduos biológicos (Q1), químicos (Q2), perfurocortantes $(\mathrm{Q} 3)$ e comuns $(\mathrm{Q} 4)$, de acordo com o questionário, observa-se que a maioria dos servidores entrevistados têm conhecimento do manejo na segregação dos resíduos químicos (74\%), resíduos biológicos (76\%) e com destaque para os resíduos perfurocortantes (100\%).

Foi observado também na pesquisa de Gomes et al. (2015) que os resíduos perfurocortantes foram segregados de forma correta segundo $100 \%$ entrevistados. Quanto às respostas a respeito do manejo dos resíduos: acondicionamento dos resíduos biológicos (Q5), químicos (Q6), perfurocortantes (Q7), resíduos comuns (Q8), conhecimento da identificação por símbolos (Q9) e armazenamento de mercúrio dos resíduos de amalgama (Q18). Observa-se que a forma adequada sobre o manejo no acondicionamento dos resíduos não é do conhecimento de todos os entrevistados. Segundo $82 \%$ das respostas dos entrevistados, os resíduos tipo A deveriam ser acondicionados em sacos plásticos, mas as cores não foram definidas.

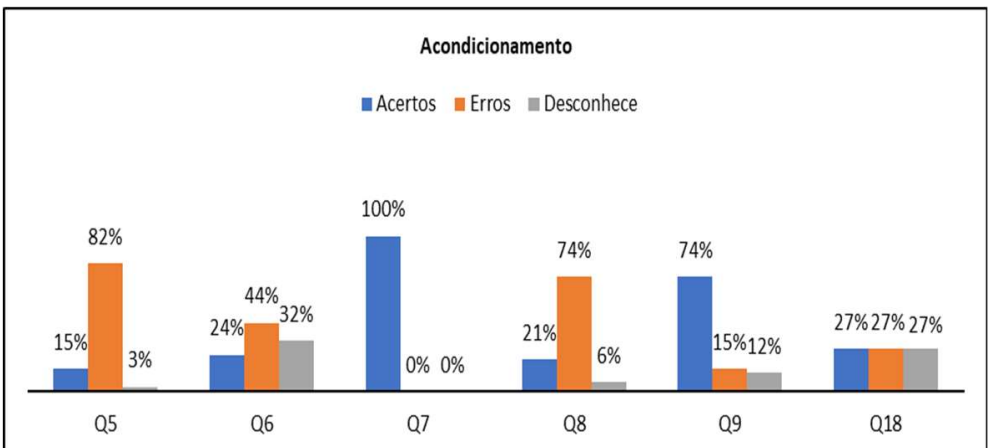

Figura 4: Percentual da percepção dos profissionais sobre o manejo dos resíduos gerados no acondicionamento dos resíduos.

Com relação aos resíduos químicos: acondicionamento do mercúrio, resíduos do amálgama, respondida apenas pelos cirurgiões dentistas e ASB, $27 \%$ responderam de maneira correta ao afirmar que devem ser armazenados em recipiente inquebrável, longe do calor, com selo d'água e símbolo de substância tóxica e expressão: 'resíduo químico'. Estando de acordo com Art. 69 RDC n 222/2018. Em relação ao erro relacionado ao armazenamento do mercúrio do resíduo do amalgama (Q18) foi de $27 \%$, ao responderem que devem ser armazenados em recipientes fechado de vidro, em local arejado e identificados com rótulos simbolizados para substâncias tóxicas. Já o artigo 69, parágrafo único da Resolução da Diretoria Colegiada ${ }^{\circ}$ 222 de 2018, determina que os RSS que contêm mercúrio (Hg) na forma líquida, devem ser acondicionados em recipientes com selo d'água e encaminhados para recuperação ou outra destinação que estejam de acordo com as regras definidas pelo órgão ambiental competente. A respeito dos resíduos perfurocortantes, todos tinham conhecimento de como acondicioná-los corretamente (100\%). A percepção a respeito da identificação por símbolos, $74 \%$ responderam de forma correta.

De acordo com Art. 15 da RDC 222/2018 os resíduos de serviço de saúde do grupo A (Resíduos biológicos) devem ser acondicionados em sacos plásticos de cor brancos-leitoso. Já na mesma RDC 222/2018 Art. 56 o gerenciamento dos RSS do Grupo B deve observar a periculosidade das substâncias presentes, 
decorrentes das características de inflamabilidade, corrosividade, reatividade e toxidade. Estudo sobre avaliação dos resíduos de medicamento e outros RSS na Região Serrana de Santa Catarina, avaliou-se que apesar do avanço nos aspectos legislativos a respeito da gestão $O$ grupo $E$ (perfurocortantes) é identificado pelos símbolos de substâncias infectante constante na NBR-7500 da ABNT, com rótulos de fundo branco, desenho e contornos pretos, acrescido da inscrição de RESÍDUOS PERFUROCORTANTE, indicando o risco que apresenta o resíduo. dos RSS, ainda há grande deficiência no descarte de resíduos medicamentos, constituindo um grande desafio para o poder público (AMARANTE et al., 2017).

O artigo 69, parágrafo único da Resolução da Diretoria Colegiada n 222 de 2018, determina que os RSS que contêm mercúrio $(\mathrm{Hg})$ na forma líquida, devem ser acondicionados em recipientes com selo d'água e encaminhados para recuperação ou outra destinação que estejam de acordo com as regras definidas pelo órgão ambiental competente. Os resíduos de serviços de saúde (RSS) oriundos da prática odontológica merecem atenção em seu gerenciamento, pois muitos são materiais altamente contaminantes gerando riscos, agravos e acidentes nas atividades, principalmente os avaliados pelo presente estudo que são os materiais comuns na prática clínica, tais como: resíduo de mercúrio do amálgama, revelador e fixador radiográfico (GARBIN et al., 2015).

Segundo Mondelli (2014) o gerenciamento e descarte adequados dos resíduos químicos de mercúrio do amálgama, não gera prejuízo significativo à saúde e ao meio ambiente. Os resíduos do grupo $\mathrm{E}$ devem ser descartados em embalagens com paredes rígidas, resistentes a perfuração e devidamente identificadas, mas esse resíduo não pode transbordar, sendo o correto serem preenchidas até $2 / 3$ do seu tamanho, segundo a RDC 222 /2018 (ANVISA, 2018).

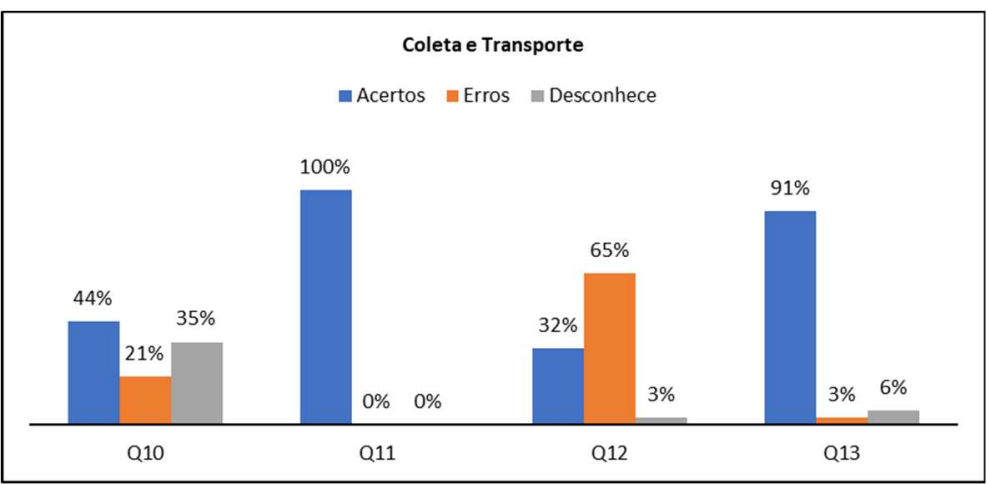

Figura 5: Percentual das respostas relacionadas à coleta e transporte dos resíduos de serviços de saúde gerados.

A RDC n 222/2018, em seu Artigo 22, determina que a identificação dos RSS deve estar afixada nos carros de coleta, nos locais de armazenamento e nos sacos que acondicionam os resíduos. Com relação à forma utilizada para transportar os resíduos do local gerado até onde é efetuada a coleta externa (Q10), se a coleta externa é feita por empresa especializada (Q11), frequência desta coleta (Q12) e local de armazenamento dos resíduos gerados (Q13), os resultados apresentados na Figura 5.

De maneira geral, os dados levantados apontam para uma maior compreensão a respeito da existência de um local exclusivo para armazenamento dos resíduos gerados, com 91\% das respostas afirmativas. Apenas $44 \%$ dos funcionários responderam que a forma de transportar o resíduo do local gerado 
até o local da coleta externa deve ser realizado em carrinho com tampa. No artigo 26 da RDC 222/2018, determina que quanto ao transporte interno, deve ser constituído de material liso, rígido, lavável, impermeável, provido de tampa articulada ao próprio corpo do equipamento, cantos e bordas arredondados. Apenas 32\% responderam de forma correta a frequência da coleta deveria ser de uma vez por semana. A existência de uma empresa especializada para coleta externa era de conhecimento de todos, com $100 \%$ das respostas afirmativas.

No trabalho realizado sobre o Gerenciamento de resíduos de serviço de saúde no Hospital do Rio de Janeiro, foi observado que o transporte interno dos resíduos infectantes era transportado nos mesmos recipientes que os resíduos comuns (PEREIRA et al., 2017). Szczerbowski et al. (2017) destacaram a eficiência da coleta dos resíduos do grupo A, B e E utilizavam veículos e profissionais devidamente preparados e equipados por empresa especializada, coletando os resíduos na unidade geradora seguindo as normas da ABNT na Unidade Básica de Saúde da Cidade de estrela do Sul, em Minas Gerais.

Quanto a destinação final dos resíduos gerados, 62\% dos funcionários afirmaram que a destinação final dos resíduos gerados no hospital e unidades de saúde são os aterros sanitários (Q14). Enquanto 6\% dos entrevistados responderam, a respeito da destinação final, que acreditavam que era feita em aterro industrial ou queimados. Entretanto 32\% relataram não saber qual era o destino dos resíduos de serviço de saúde.

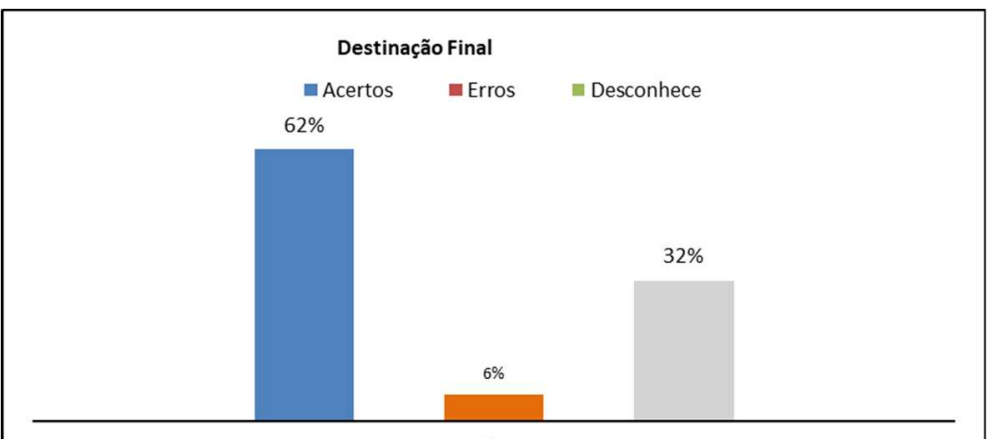

Figura 6: Percentual das respostas relacionadas à destinação Final dos resíduos gerados nos serviços de saúde.

O parágrafo 5으, do artigo 46 da $\operatorname{RDC} \mathrm{n}^{\circ} 222 / 2018$, determina que os rejeitos devem ser encaminhados para disposição final ambientalmente adequada, após o tratamento. Quanto às respostas relacionadas à reciclagem dos resíduos gerados, os dados levantados mostram uma variação de 47-65\% nos acertos sobre o conhecimento do encaminhamento para reciclagem. As questões neste item foram: encaminhamento de resíduos para reciclagem (Q15), resíduos oriundos da radiologia (Q16), resíduos radiológicos passíveis de reciclagem (Q17) e quanto ao descarte de soluções reveladoras e fixadoras das películas dos filmes radiográficos (Q19). Já 53\% dos entrevistados responderam que soluções processadoras, chumbo, prata e películas dos filmes radiográficos são oriundas da radiologia e devem ser reciclados. A maior conformidade está relacionada ao descarte de soluções fixadoras das películas dos filmes radiográficos, com $65 \%$ dos acertos, que deve ser acondicionado até a coleta no local de trabalho e encaminhadas para reciclagem. Embora $47 \%$ dos servidores soubessem da possibilidade de reciclar os resíduos radiológicos contendo prata e base de poliéster dos exames radiológicos descartados, a questão Q15 mostra que nenhum material destas 
unidades segue para reciclagem.

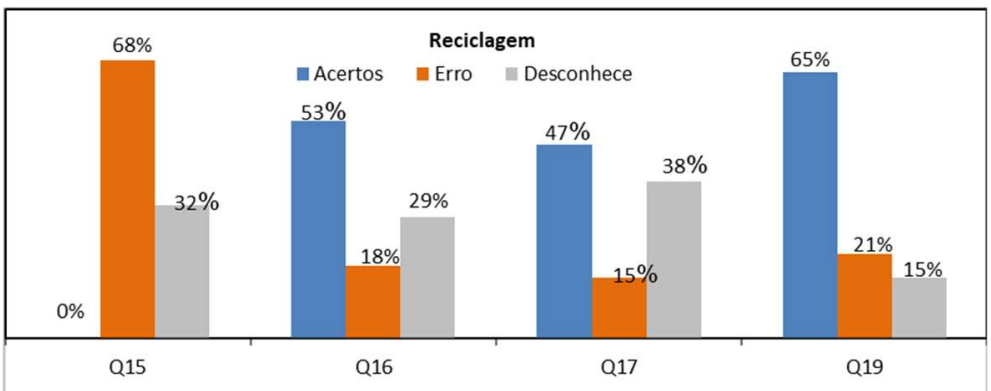

Figura 7: Percentual das respostas relacionadas à reciclagem dos resíduos de serviços de saúde.

De acordo com o artigo 40 da RDC n²22/2018, RSS que não apresentam risco biológico, químico ou radiológico, podem ser encaminhados para reciclagem, recuperação, reutilização, compostagem, aproveitamento energético ou logística reversa.

\section{CONCLUSÕES}

Este Trabalho abordou a relevância das questões importantes dos RSS, sendo uma problemática que afeta diretamente a Saúde Pública, tornando-se ainda necessário a constante discussão sobre este tema entre todos os atores sociais envolvidos e as instituições de Saúde do município. Ficou demonstrado a dificuldade da maioria dos profissionais com relação ao conhecimento do Plano de Gerenciamento de Resíduos de Serviço de Saúde - PGRSS quando 47\% desconhece a existência do documento elaborado pelo município, contudo, 35\% disseram existir o PGRRS, embora este não tenha sido apresentado durante o desenvolvimento da pesquisa.

No manejo e segregação dos resíduos gerados, $82 \%$ dos profissionais tem domínio neste requisito, entretanto na questão de acondicionamento dos resíduos apenas $43 \%$ tem conhecimento, o que é insuficiente dadas às proporções e relevâncias das questões ambientais envolvidas. Todos os profissionais relataram que a coleta final dos RSS é realizada por empresa especializada.

No âmbito geral, o profissional de saúde, do serviço público do município da Zona da Mata de Pernambuco: Hospital Geral e Unidades Básicas de Saúde têm conhecimento sobre gestão de resíduos de serviços de saúde de acordo com as exigências da RDC n 222/2018 em torno de 54\% do total. Um dos aspectos a ser melhorado no PGRSS seria um reforço na Educação Ambiental e Sanitária dos profissionais dos serviços de saúde. Os profissionais conscientes da importância da melhoria do meio ambiente e da garantia da própria saúde ocupacional oferecerão melhores serviços à sociedade e um atendimento seguro, portanto sugere-se a realização de outros estudos com as demais categorias profissionais que atuam no hospital e unidades básicas de saúde para efetivação do PGRSS.

\section{REFERÊNCIAS}

ANVISA. Agência Nacional de Vigilância Sanitária. RDC n.222/2018. Brasília: ANVISA, 2018.

ABRELPE. Associação Brasileira de Empresas de Limpeza Pública e Resíduos Especiais. Panorama de Resíduos Sólidos no Brasil 2017. São Paulo: ABRELPE, 2018.
ALAM, I.; ALAM, G.; AYUB, S.; SIDDIQUI, A. A.. Assessment of Bio-medical Waste Management in Different Hospitals in Aligarh City. In: Advances in Waste Management. Singapore: Springer Singapore, 2019. p.501-510. 
ALVES, A. K. S.; ARAÚJO, A. A.; RIBEIRO, Í. A. P.; DUARTE NETA, M. A.; LAGES, M. G. G.; RIBEIRO, R. O.. Gestão dos resíduos de serviços de saúde: mitigação dos impactos sanitário e ambiental. Revista Uningá Review, v.25, n.2, p.66-72, 2016.

AMARANTE, J. A. S.; RECH, T. D.; SIEGLOCH, A. E.. Avaliação do gerenciamento dos resíduos de medicamentos e demais resíduos de serviços de saúde na Região Serrana de Santa Catarina, Brasil. Engenharia Sanitária Ambiental, v.22, n.2, p.317-326, 2017.

CONAMA. Conselho Nacional do Meio Ambiente. Resolução no 358, de 29 de abril de 2005. Dispõe sobre o tratamento e a disposição final dos resíduos dos serviços de saúde e dá outras providências. Brasília: CONAMA, 2005.

CAFURE, V. A.; PATRIARCHA-GRACIOLLI, S. R.. Os resíduos de serviço de saúde e seus impactos ambientais: uma revisão bibliográfica. Interações, Campo Grande, v.16, n.2, p.301314, 2015.

GARBIN, A. J. I.; WAHAYAMA, B.; BRTTO, C. P.; GARBIN, C. A. S.. A imperícia no gerenciamento dos Resíduos de Serviços de Saúde nos consultórios odontológicos privados. Archives of Health Investigation, v.4, n.5, p.1-5, 2015.

GOMES, P. A. M.; GARBIN, A. J. I.; ARCIERI, R. M.; ROVIDA, T.
A.; GARBIN, C. A. S.. Saúde e segurança no trabalho: as implicações do processo de gerenciamento dos resíduos de saúde no serviço público. Archives of Health Investigation, v.4, n.4, p.44-49, 2015.

KHOBRAGADE, D. S.. Health care waste: avoiding haszards to living and nonliving environment by efficient management. Fortune J Health Sci., v.2, n.2, p.14-29, 2019.

LEVIN, J.. Estatística aplicada a ciências humanas. São Paulo: Harbra, 1987.

MONDELLI, J.. O que o cirurgião-dentista que prática a Odontologia deve saber a respeito do amálgama dentário. Full Dent. Sciense, v.5, n.19, p.511-526, 2014.

PEREIRA, S. V.; MORAES, M. J. C. P.; VEIGA, L. B. E.. Gerenciamento de serviço de saúde, desafios e ecoeficiência: estudo de caso em um Hospital no Rio de Janeiro. In: CONGRESSO ENGEMA, 19. Anais. 2017.

SZCZERBOWSKI, A. C.; MORAIS, C. R.. Manejo de resíduos sólidos em unidade básica de saúde da cidade de Estrela do Sul, Minas Gerais, Brasil. Getec, v.6, n.11, p.29-40, 2017.

TRIOLA, M. F.. Introdução à Estatística. 10 ed. Rio de Janeiro: LTC, 2008. 